\title{
What is brain health and why is it important?
}

\section{Yongjun Wang and colleagues discuss the definition of brain health and the opportunities and challenges of future research}

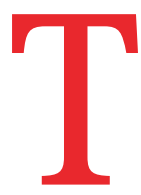

he human brain is the command centre for the nervous system and enables thoughts, memory, movement, and emotions by a complex function that is the highest product of biological evolution. Maintaining a healthy brain during one's life is the uppermost goal in pursuing health and longevity. As the population ages, the burden of neurological disorders and challenges for the preservation of brain health increase. It is therefore vital to understand what brain health is and why it is important. This article is the first in a series that aims to define brain health, analyse the effect of major neurological disorders on brain health, and discuss how these disorders might be treated and prevented.

\section{Definition of brain health}

Currently, there is no universally recognised definition of brain health. Most existing definitions have only a general description of normal brain function or emphasise one or two dimensions of brain health. The US Centers for Disease Control and Prevention defined brain health as an ability to perform all the mental processes of cognition, including the ability to learn and judge, use language, and remember. ${ }^{1}$ The American Heart Association/American Stroke Association (AHA/ASA) presidential advisory defined optimal brain health as "average performance levels among all people at that age who are free of known brain or other organ system diseases in terms of decline

\section{KEY MESSAGES}

- Brain health is the preservation of optimal brain integrity and mental and cognitive function and the absence of overt neurological disorders

- Human ageing increases the burden of brain dysfunction and neurological diseases and the demands for medical resources

- Further studies are required to assess brain health, understand the mechanism of brain function and dysfunction, and explore effective approaches to promote brain health. from function levels, or as adequacy to perform all activities that the individual wishes to undertake." 2

The brain is a complex organ and has at least three levels of functions that affect all aspects of our daily lives: interpretation of senses and control of movement; maintenance of cognitive, mental, and emotional processes; and maintenance of normal behaviour and social cognition. Brain health may therefore be defined as the preservation of optimal brain integrity and mental and cognitive function at a given age in the absence of overt brain diseases that affect normal brain function.

\section{Effect of major neurological disorders on brain health}

Several neurological disorders may disrupt brain function and affect humans' health. Medically, neurological disorders that cause brain dysfunction can be classified into three groups:

- $\quad$ Brain diseases with overt damage to brain structures, such as cerebrovascular diseases, traumatic brain injury, brain tumours, meningitis, and communication and sensory disorders

- Functional brain disorders with detectable destruction of brain connections or networks, such as neurodegenerative diseases (eg, Parkinson's disease, Alzheimer's disease, and other dementias) and mental disorders (eg, schizophrenia, depression, bipolar disorder, alcoholism, and drug abuse)

- Other brain disorders without detectable structural or functional impairment, such as migraine and sleep disorders.

These neurological disorders may have different or common effects on brain health and function. For instance, Alzheimer's disease is the main type of dementia, with a decline in different domains of cognitive function. Mood disorders may cause dysfunction in execution, reward processing, and emotional regulations. In addition to physical disability, aphasia, gait and balance problems, and cerebrovascular diseases may lead to cognitive impairment and dementia, which are neglected by both patients and physicians.

\section{Ageing and burden of neurological disorders}

Human ageing is mainly reflected in the aspects of brain ageing and degradation of brain function. The number of people aged 60 years and over worldwide was around 900 million in 2015 and is expected to grow to two billion by $2050 .{ }^{3}$ With the increases in population ageing and growth, the burden of neurological disorders and challenges to the preservation of brain health steeply increase. People with neurological disorders will have physical disability, cognitive or mental disorders, and social dysfunction and be a large economic burden.

Globally, neurological disorders were the leading cause of disability adjusted life years (276 million) and the second leading cause of death (9 million) in 2016, according to the Global Burden of Diseases study. ${ }^{4}$ Stroke, migraine, Alzheimer's disease and other dementias, and meningitis are the largest contributors to neurological disability adjusted life years. ${ }^{4}$ About one in four adults will have a stroke in their lifetime, from the age of 25 years onwards. ${ }^{5}$ Roughly 50 million people worldwide were living with dementia in 2018, and the number will more than triple to 152 million by $2050 .^{6}$ In the following decades, governments will face increasing demand for treatment, rehabilitation, and support services for neurological disorders.

\section{Opportunities and challenges of future research on brain health}

Opportunities and challenges exist in the assessment of brain health, the mechanism of brain function and dysfunction, and approaches to promote brain health (box 1).

Defining and promoting optimal brain health require the scientific evaluation of brain health. However, it is difficult to comprehensively evaluate or quantify brain health through one metric owing to the multidimensional aspects of brain health. Many structured or semistructured questionnaires have been developed to test brain health by self-assessments or close family member assessments of daily function or abilities. In recent decades new structural and functional neuroimaging techniques have been applied to evaluate brain network integrity and functional 


\section{Box 1: Opportunities and challenges of future research on brain health}

- Lack of metrics or tools to comprehensively assess or quantify brain health

- Little knowledge about the mechanisms of brain function and dysfunction

- Few effective approaches to prevent and treat brain dysfunction in some major neurological disorders, such as dementia

- Need to precisely preserve brain functions for people with neurosurgical diseases

connectivity. ${ }^{7}$ However, these subjective or objective measures have both strengths and weaknesses. For instance, scales such as the mini-mental state examination and Montreal cognitive assessment are simple and easy to implement but are used only as global screening tools for cognitive impairment; tests such as the digit span, Rey-Osterrieth complex figure test, trail making A and B, Stroop task, verbal fluency test, Boston naming test, and clock drawing test are used mainly to assess one or two specific domains of memory, language, visuospatial, attention, and executive function; and neuroimaging techniques, although non-invasive and objective, still have disadvantages of test contraindications, insufficient temporal or spatial resolution, motion artefact, and high false discovery rates, which limit their clinical transformation.

Another difficulty in measuring brain health is that age, culture, ethnicity, and geography specific variations exist in the perception of optimal brain health. Patient centred assessment of brain function, such as self-perception of cognitive function and quality of life, should also be considered when measuring brain health. ${ }^{8}$ Universal acceptable, age appropriate, multidimensional, multidisciplinary, and sensitive metrics or tools are required to comprehensively measure and monitor brain function and brain health.

To promote optimal brain health, we need a better understanding of the mechanisms of brain function and dysfunction. Unfortunately, little is known about the working mechanism of the brain. Although we have made considerable developments in neuroscience in recent decades, we still cannot totally decipher the relations between spatiotemporal patterns of activity across the interconnected networks of neurons and thoughts or the cognitive and mental state of a person. ${ }^{9}$ Recent progress in brain simulation and artificial intelligence provides a vital tool to understand biological brains, and vice versa. ${ }^{1011}$ The development of brain inspired computation, brain simulation, and intelligent machines was emphasised in the European Union and China Brain Project. ${ }^{912}$
Meanwhile, the mechanisms behind the brain dysfunction in some neurological disorders are still not well understood, especially for mental and neurodegenerative disorders. Further investigation of the mechanisms of brain diseases may indicate approaches to treatment and improve brain function. Brain imaging based cognitive neuroscience may unravel the underlying brain mechanism of cognitive dysfunction and provide an avenue to develop a biological framework for precision biomarkers of mood disorders. ${ }^{13}$

Most common neurological diseases, such as cerebrovascular diseases and Alzheimer's disease, have complex aetiopathologies, typically involving spatial-temporal interactions of genetic and environmental factors. However, a single genetic factor could account for the disease progression of monogenic neurological disorders. These diseases could be more readily investigated by simplified cross species modelling, leading to better understanding of their mechanisms and greater efficiency in testing innovative therapies. Such research may provide a window to promote the investigation of common neurological disorders and general brain health, as discussed by Chen and colleagues elsewhere in this series. ${ }^{14}$

Few effective approaches are available to prevent and treat brain dysfunction in some major neurological disorders, such as dementia. Neurons are not renewable, and brain dysfunction is always irreversible. Recent trials targeting amyloid clearance and the selective inhibition of tau protein aggregation failed to improve cognition or modify disease progression in patients with mild Alzheimer's disease. ${ }^{15}{ }^{16}$ More attention has focused on other potential therapeutic targets, such as vascular dysfunction, inflammation, and the gut microbiome, as discussed by Shi and colleagues. ${ }^{17}$ In particular, recent studies showed that the early impairment of cognition was induced by the disruption of neurovascular unit integrity, which may cause hypoperfusion and the breakdown of the blood-brain barrier and subsequent impairment in the clearance of proteins in the brain. ${ }^{1819}$ Physical activity, mental exercise, a healthy diet and nutrition, social interaction, ample sleep and relaxation, and control of vascular risk factors are considered six pillars of brain health. The AHA/ASA presidential advisory recommended the AHA's Life's Simple 7 (non-smoking, physical activity, healthy diet, appropriate body mass index, blood pressure, total cholesterol, and blood glucose) to maintain optimal brain health. ${ }^{2}$ Pan and colleagues discuss how this may indicate a new dawn of preventing some cognitive impairment and brain dysfunction by preventing vascular risk factors or cerebrovascular diseases. ${ }^{20}$

For other neurological disorders with potential therapeutic approaches, the main aim is to preserve brain function. Impaired brain function due to anatomical structural damage is underestimated in patients with neurosurgical diseases such as brain tumours, trauma, and epilepsy. In recent years, treatment targets for neurosurgical diseases have changed from focusing on survival or life expectancy to balancing brain structures and functions. Precise preservation of brain function requires an understanding of the exquisite relation between brain structure and function and advanced technologies to visualise brain structure-function relations. ${ }^{21}$

Another example of the predicament associated with protection of brain function is uncertainty in the treatment response in epilepsy management. Current standard care for epilepsy relies on a trial and error approach of sequential regimens of antiseizure medications. The time delay due to this treatment approach means that such treatments may be less effective and irreversible damage may occur. Chen and colleagues $^{22}$ describe how recent advances in personalised epilepsy management based on artificial intelligence, genomics, and patient derived stem cells are bringing some hope to overcome this predicament in epilepsy management and promise a more effective strategy. ${ }^{23} 24$

Brain health is the maintenance of multidimensional aspects of brain function. However, several neurological disorders may affect brain health in one or more aspects of brain function. Deciphering and promoting the function and health of the brain, the most mysterious organ in the human body, will have a dramatic impact on science, medicine, and society. ${ }^{25}$ In the past seven years, a number of large scale brain health initiatives have been launched in several countries to promote the development of neuroscience, brain 
simulation, and brain protection. ${ }^{9}$ However, further challenges are raised by the different key research directions of brain projects in different countries. In the face of these challenges, Liu and colleagues argue that collaboration on brain health research is urgently needed. ${ }^{26}$ As the other articles in this series describe, coordinated research has enormous potential to improve the prognosis of brain disorders.

Contributors and sources: YW proposed the idea for this series on brain health. YW and YP drafted the first manuscript. All the authors critically reviewed and revised the manuscript. YP and $\mathrm{HL}$ expertise is in the area of clinical research methods and clinical research on stroke. YW is an expert in clinical research on stroke and neurological diseases. YW is the guarantor.

Competing interests We have read and understood BMJ policy on declaration of interests and declare that the study was supported by grants from the National Science and Technology Major Project (2017ZX09304018), National Key R\&D Program of China (2018YFC1312903, 2017YFC1310902, 2018YFC1311700, and 2018YFC1311706), National Natural Science Foundation of China (81971091), Beijing Hospitals Authority Youth Programme (QML20190501), and Beijing Municipal Science and Technology Commission (D171100003017002).

Provenance and peer review: Commissioned; externally peer reviewed.

This article is part of a series launched at the Chinese Stroke Association annual conference on 10 October 2020, Beijing, China. Open access fees were funded by the National Science and Technology Major Project. The BM/ peer reviewed, edited, and made the decision to publish these articles.

Yongjun Wang, professor ${ }^{1,2}$

Yuesong Pan, associate professor ${ }^{1,2}$

Hao Li, professor ${ }^{1,2}$

${ }^{1}$ Department of Neurology, Beijing Tiantan Hospital, Capital Medical University, Beijing, China

${ }^{2}$ China National Clinical Research Center for Neurological Diseases, Beijing, China

Correspondence to: YWang yongjunwang@ncrend.org.cn

\section{(c) (1) () OP OPEN ACCESS}

This is an Open Access article distributed in accordance with the Creative Commons Attribution Non Commercial (CC BY-NC 4.0) license, which permits others to distribute, remix, adapt, build upon this work non-commercially, and license their derivative works on different terms, provided the original work is properly cited and the use is non-commercial. See: http://creativecommons.org/ licenses/by-nc/4.0/.

\section{Check for updates}

1 Centers for Disease Control and Prevention. Healthy aging. What is a healthy brain? New research explores perceptions of cognitive health among diverse older adults. https://www.cdc.gov/aging/pdf/ perceptions of cog_hlth_factsheet.pd

2 Gorelick PB, Furie KL, Iadecola C, et al, American Heart Association/American Stroke Association. Defining optimal brain health in adults: a presidential advisory from the American Heart Association/ American Stroke Association. Stroke 2017;48:e284303. doi:10.1161/STR.0000000000000148

3 WHO Global Health Ethics team. Ageing. https:// www.who.int/ethics/topics/ageing/en/. 2019

4 GBD 2016 Neurology Collaborators. Global, regional, and national burden of neurological disorders, 1990 2016: a systematic analysis for the Global Burden of Disease Study 2016. Lancet Neurol 2019;18:45980. doi:10.1016/S1474-4422(18)30499-X

5 Feigin VL, Nguyen G, Cercy K, et al, GBD 2016 Lifetime Risk of Stroke Collaborators. Global, regional, and country-specific lifetime risks of stroke, 1990 and 2016. N Engl I Med 2018;379:2429-37. doi:10.1056/NEJMoa1804492

6 Alzheimer's Disease International. World Alzheimer report 2018. The state of the art of dementia research: new frontiers. https://www.alz.co.uk/ research/world-report-2018

7 Modi HN, Singh H, Yang GZ, Darzi A, Leff DR. A decade of imaging surgeons' brain function (part I): Terminology, techniques, and clinical translationSurgery 2017;162:1121-30 doi:10.1016/j.surg.2017.05.021

8 Gordon MF, Lenderking WR, Duhig A, et al, PatientReported Outcome Consortium's Cognition Working Group. Development of a patient-reported outcome instrument to assess complex activities of daily living and interpersonal functioning in persons with mild cognitive impairment: The qualitative research phase. Alzheimers Dement 2016;12:75-84 doi:10.1016/j.jalz.2015.04.008

9 Yuste R, Bargmann C. Toward a Global BRAIN Initiative. Cell 2017;168:956-9. doi:10.1016/j. cell.2017.02.023

10 Lake BM, Ullman TD, Tenenbaum JB, Gershman SJ. Building machines that learn and think like people. Behav Brain Sci 2017:40:e253. doi:10.1017/ S0140525X16001837

11 Hassabis D, Kumaran D, Summerfield C, Botvinick M. Neuroscience-inspired artificial intelligence. Neuron 2017;95:245-58. doi:10.1016/j. neuron.2017.06.011
12 Poo MM, Du JL, Ip NY, Xiong ZO, Xu B, Tan T. China Brain Project: basic neuroscience, brain diseases, and brain-inspired computing. Neuron 2016;92:591-6. doi:10.1016/j. neuron.2016.10.050

13 Chen R, Cui Z, Capitao L, et al. Precision biomarkers for mood disorders based on brain imaging. BMJ 2020;371:m3618.

14 Chen W-J, Cheng X, Fu Y, et al. Rethinking monogenic neurological diseases. BMJ 2020;371:m3752.

15 Doody RS, Thomas RG, Farlow M, et al, Alzheimer's Disease Cooperative Study Steering Committee, Solanezumab Study Group. Phase 3 trials of solanezumab for mild-to-moderate Alzheimer's disease. N Engl J Med 2014;370:311-21. doi:10.1056/NEIMoa1312889

16 Gauthier S, Feldman HH, Schneider LS, et al. Efficacy and safety of tau-aggregation inhibitor therapy in patients with mild or moderate Alzheimer's disease: a randomised, controlled, double-blind, parallelarm, phase 3 trial. Lancet 2016;388:2873-84. doi:10.1016/S0140-6736(16)31275-2

17 Shi J, Sabbagn MN, Vellas B. Alzheimer's disease beyond amyloid: strategies for future therapeutic interventions. BMI 2020:371:m3684.

18 Henstridge CM, Hyman BT, Spires-Jones TL. Beyond the neuron-cellular interactions early in Alzheimer disease pathogenesis. Nat Rev Neurosci 2019;20:94-108. doi:10.1038/s41583018-0113-1

19 Sweeney MD, Kisler K, Montagne A, Toga AW, Zlokovic BV. The role of brain vasculature in neurodegenerative disorders. Nat Neurosci 2018;21:1318-31. doi:10.1038/s41593018-0234-X

20 Pan Y, Li H, Wardlaw JM, Wang Y. A new dawn of preventing dementia by preventing cerebrovascular diseases. BMJ 2020;371:m3672.

21 Zhang L, Li D, Xiao D, Couldwell WT, Ohata K. Improving brain health by identifying structure function relations in patients with neurosurgical disorders. BM/ 2020.371:m3690.

22 Chen Z, Rollo B, Antonic-Baker A, et al. New era of personalised epilepsy management. BM/ 2020:371:m3658.

23 Kuhlmann L, Lehnertz K, Richardson MP, Schelter B, Zaveri HP. Seizure prediction - ready for a new era. Nat Rev Neurol 2018:14:618-30. doi:10.1038/ s41582-018-0055-2

24 Epi4K Consortium. Epi4K: gene discovery in 4,000 genomes. Epilepsia 2012:53:1457-67. doi:10.1111/j.1528-1167.2012.03511.x

25 Wang DZ, Schwamm LH, Qian T, Dai Q. Decoding the brain through research-the future of brain health. BMJ 2020;371:m3735.

26 Liu L, Feigin V, Sacco RL, Koroshetz WJ. Promoting global collaboration for brain health research. BMJ 2020;371:m3753.

Cite this as: BMJ 2020;371:m3683

http://dx.doi.org/10.1136/bmj.m3683 